\title{
Convolutional Neural Networks for Direct Text Deblurring
}

\section{Michal Hradiš ${ }^{1}$}

ihradis@fit.vutbr.cz

Jan Kotera ${ }^{2}$

kotera@utia.cas.cz

Pavel Zemčík ${ }^{1}$

zemcik@fit.vutbr.cz

Filip Šroubek ${ }^{2}$

sroubekf@utia.cas.cz

\author{
${ }^{1}$ Faculty of Information Technology \\ Brno University of Technology \\ Brno, Czech Republic \\ ${ }^{2}$ Institute of Information Theory and Automation \\ Czech Academy of Sciences \\ Prague, Czech Republic
}

We investigate if convolutional neural networks $(\mathrm{CNN})$ can learn to directly perform blind image deconvolution and restoration - that is if they can provide high-quality restored images directly from blurred inputs without any knowledge of the specific degradataion process. In our experiments on text documents, CNNs significantly outperformed existing blind deconvolution methods, including those optimized for text, in terms of image quality and OCR accuracy. In fact, the convolutional networks outperformed even state-of-the-art non-blind methods for anything but the lowest noise levels.

The architectures we use are inspired by the very successful networks that recently redefined state-of-the-art in many computer vision tasks starting with image classification on ImageNet by Krizhevsky et al. [2]. The networks are composed of multiple layers of convolutions and element-wise Rectified Liear Units (ReLU):

$$
\begin{aligned}
& F_{0}(y)=y \\
& F_{l}(y)=\max \left(0, W_{l} * F_{l-1}(y)+b_{l}\right), l=1, \ldots, L-1 \\
& F(y)=W_{L} * F_{L-1}(y)+b_{L}
\end{aligned}
$$

The input and output are both 3-channel RGB images with values mapped to interval $[-0.5,0.5]$. Each layer applies $c_{l}$ convolutions with filters spanning all channels $c_{l-1}$ of the previous layer. The last layer is linear (without ReLU).

As in previous works [5], we train the networks by minimizing mean squared error on a dataset $D=\left(x_{i}, y_{i}\right)$ of corresponding clean and corrupted image patches:

$$
\underset{W, b}{\arg \min } \frac{1}{2|D|} \sum_{\left(x_{i}, y_{i}\right) \in D}\left\|F\left(y_{i}\right)-x_{i}\right\|_{2}^{2}+0.0005\|W\|_{2}^{2}
$$

We evaluated the approach on a large set of documents from the CiteSeerX repository which we rendered at 120-150 DPI. A dataset was created by aplying random geometric transformations simulating deviations from optimal camera position to small rendered page regions and by convolving with realistic de-focus and camera-shake blur kernels (distribution of kernel sizes is show in Figure 1 right-bottom). We purposely limited the image degradations to shift-invariant blur and additive noise to allow for fair comparison with the baseline methods, which are not designed to handle other aspects of an imaging process.

In our experiments, deeper networks performed singnificantly better and restoration quality was fairly insensitive to other architectural choices. This can be clearly seen in Figure 1. Top-left shows best the benefit of
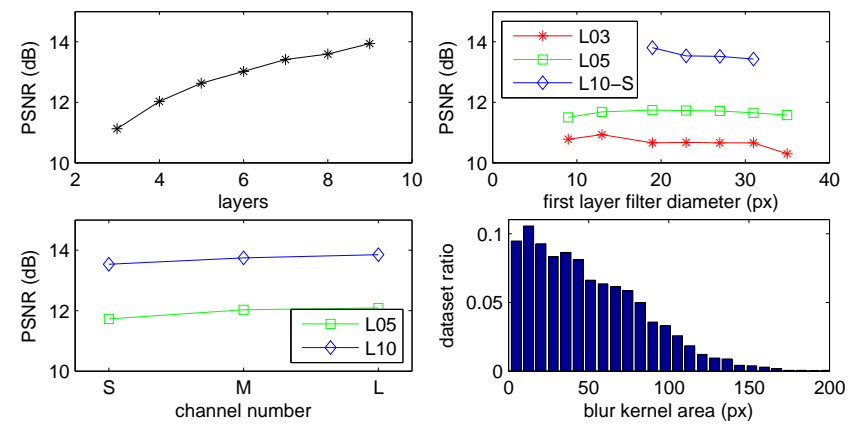

Figure 1: Different CNN architectures. The number after L (e.g. L10) indicates number of layers in the network. top-left - network depth; topright - spatial support size; bottom-left - channel number; bottom-right distribution of blur-kernel sizes in dataset

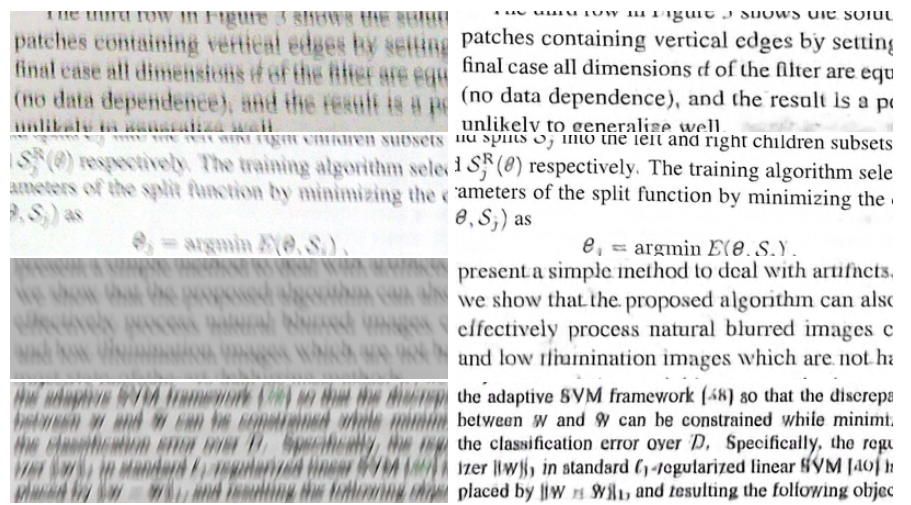

Figure 2: $\mathrm{CNN}$ deblurring results of challenging real images.

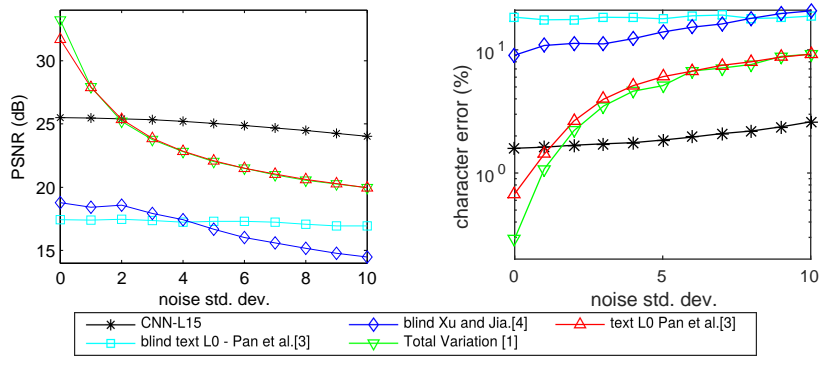

Figure 3: Blind deconvolution image quality (left) and OCR error on reconstructed images (right).

deeper networks. Top-right shows that performance is insensitive to the size of the first layer filters. Bottom-left shows that networks with more channels per layer perform slightely better, but the benefit is negligable compared to increased network depth.

The largest and deepest network we trained has 15 layers with $2.3 \mathrm{M}$ parameters (PSNR $16.06 \mathrm{~dB}$ on the dataset from Figure 1). We compared this network with two blind [3, 4] and two non-blind [1,3] methods on $200 \times 200$ image patches extracted from unseen documents (see Figure 3 ). The methods of Pan et al. [3] were designed specifically for text images. The CNN clearly outperforms the blind methods for all noise levels and the non-blind methods for all but the lowest noise levels. Surprisingly, the $\mathrm{CNN}$ maintains good quality even for noise levels higher than for what it was trained for.

[1] Stanley H. Chan, Ramsin Khoshabeh, Kristofor B. Gibson, Philip E. Gill, and Truong Q. Nguyen. An augmented Lagrangian method for total variation video restoration. IEEE TIP, 20(11):3097-3111, 2011.

[2] Alex Krizhevsky, Ilya Sutskever, and Geoffrey E Hinton. ImageNet Classification with Deep Convolutional Neural Networks. NIPS, 2012.

[3] Jinshan Pan, Zhe Hu, Zhixun Su, and Ming-Hsuan Yang. Deblurring Text Images via L0-Regularized Intensity and Gradient Prior. In CVPR, 2014.

[4] Li Xu and Jiaya Jia. Two-phase kernel estimation for robust motion deblurring. In $E C C V, 2010$.

[5] Li Xu, Jimmy SJ. Ren, Ce Liu, and Jiaya Jia. Deep Convolutional Neural Network for Image Deconvolution. In NIPS, 2014. 